\title{
Modélisation et ingénierie de la photosynthèse
}

\author{
Jean-François CORNET \\ Professeur en Energétique et Génie des Procédés \\ ENSCCF, Institut Pascal, UMR-CNRS/UBP/IFMA 6602 \\ Ensemble scientifique des Cézeaux - Bât. Polytech. \\ 24, avenue des Landais, 63174 AUBIERE Cedex \\ j-francois.cornet@ensccf.fr
}

Olivier BERNARD

Directeur de Recherche

BIOCORE

INRIA/INRA/CNRS/UPMC

BP93

06902 Sophia Antipolis cedex

olivier.bernard@inria.fr

La modélisation numérique a permis d'améliorer de nombreux procédés biotechnologiques à tous les niveaux (conception, optimisation, commande, supervision). C'est également le cas pour la culture des microalgues, où des efforts intenses sont aujourd'hui déployés pour développer des modèles de divers niveaux de complexité en fonction des objectifs et des dispositifs de production considérés (photobioréacteurs ou raceways), relevant cependant tous de l'ingénierie.

Lorsqu'il s'agit de produire de la biomasse, le procédé est conduit de façon à ce que tous les éléments nutritifs soient maintenus à des concentrations optimales pour la croissance. Les deux principaux facteurs restants (éminemment variables dans le temps) agissant sur la croissance sont alors la lumière et la température dont les évolutions résultent du flux d'énergie solaire.

L'évolution de la température peut être modélisée par des bilans thermiques incluant le rayonnement et dépendant fortement de l'évaporation liée à des choix technologiques de culture en systèmes ouverts ou fermés. Des modèles permettent ensuite de prévoir l'effet de la température sur la vitesse de croissance et sur certaines réactions telles que la synthèse de pigments. La principale caractéristique des modèles de cultures photosynthétiques reste alors le transfert de lumière au sein du volume réactionnel. Cet aspect est central pour au moins deux raisons : (i) c'est la principale limitation physique du procédé (comme tout procédé solaire d'ailleurs) qui fixe donc, pour une température donnée, les performances cinétiques et énergétiques des photobioréacteurs ; (ii) le champ de radiation apparaît comme la principale contrainte sur le métabolisme des micro- 
organismes (hors forçage physiologique); il est donc responsable de la qualité de la biomasse produite.

\section{Couplages entre modèles physiques et biologiques}

La prise en compte de ces facteurs et leur impact sur la croissance des micro-organismes dans le volume réactionnel implique de considérer le couplage entre des modèles physiques et biologiques. Les principaux aspects développés dans ces modèles physiques concernent la prédiction des propriétés optiques des microalgues (indices de réfraction) dont on peut déduire par des approches électromagnétiques les propriétés radiatives (figure 1) qui sont des paramètres d'entrée à la résolution de l'équation de transport de photons dans le mélange réactionnel. Cette résolution, notamment en géométrie complexe, est très difficile et on utilise aujourd'hui les dernières avancées des méthodes de Monte Carlo intégrales qui permettent d'analyser la sensibilité aux paramètres (teneur en pigments, taille des cellules, concentration en biomasse, ...), mais aussi la sensibilité au domaine qui débouche sur l'optimisation géométrique des photobioréacteurs.

Les signaux, perçus à l'échelle d'une cellule qui traverse rapidement des gradients de lumière, imposent de représenter correctement dans les modèles les mécanismes primaires de conversion de l'énergie photonique en ATP et pouvoir réducteur (transfert d'excitations au sein des photosystèmes, réactions d'oxydo-réduction sur les membranes photosynthétiques), puis leur couplage avec l'ensemble du métabolisme, dont l'énergie est fournie par l'étape précédente, et qui permet de réaliser l'acquisition de carbone, d'azote de phosphore, et de synthétiser la biomasse. Des approches très détaillées (modélisation par flux métaboliques) peuvent alors s'appuyer sur la connaissance des réactions de synthèse élémentaires. A terme, les cinétiques de ces réactions pourraient même être mises en relation avec le génome du micro-organisme. En effet, à des échelles de temps plus longues, des mécanismes d'adaptation, sous tendus par des régulations génétiques doivent être représentés dans les modèles (cf. V.7). C'est le cas de la photo-adaptation, capacité de la cellule à moduler son cortège pigmentaire en fonction de la lumière qu'elle reçoit. De même, le cycle cellulaire, qui s'appuie sur l'horloge interne et sur des événements externes (lumière, disponibilité en éléments nutritifs, ...) devra également, à terme, être intégré dans les modèles prédictifs. En effet, il induit une variation synchrone de la taille et du contenu pigmentaire des cellules, qui sont des quantités clés pour les modèles radiatifs (impactant ainsi les données d'entrée de la chaine de modélisation).

\section{Difficultés du champs de recherche}

Ces approches multi-échelles conduisent à des problématiques de modélisation qui sont parfois aujourd'hui non résolues, tant dans le domaine de la physique (calcul des propriétés radiatives de microalgues de formes complexes - voir Fig. 1 -) que de la biologie (meilleure compréhension des mécanismes biologiques à l'œuvre du génome au métabolisme), , en passant par la compréhension 
physique et thermodynamique de la photosynthèse (transfert d'excitations dans les antennes, couplages énergétiques au sein des organites cellulaires)..

Les modèles intégrant l'ensemble de ces mécanismes sont très complexes, difficiles à calibrer et à analyser. Leur utilisation pratique est limitée à la simulation, la conception et à l'optimisation des photobioréacteurs autorisant à ce stade des temps de calculs longs et pour lesquels de récents succès en intensification ont été obtenus. Pour la gestion opérationnelle des photobioréacteurs par contre, des modèles plus simples sont souvent utilisés. Ils sont plus faciles à calibrer et une analyse mathématique permet d'en déduire des stratégies de contrôle ou des systèmes de diagnostic. Ces approches permettent en particulier de calculer en temps réel les conditions opératoires optimales pour maximiser la productivité ou d'évaluer l'évolution de variables clé (teneurs intracellulaires, activités enzymatiques, ...) qui ne peuvent pas être mesurées directement.

Comme dans de nombreux autres domaines des biotechnologies, l'utilisation de modèles numériques capables de prédire de plus en plus finement la productivité d'un dispositif de culture va se généraliser. Ces outils permettront de rationnaliser et d'optimiser le design, et de maximiser la productivité dans un contexte où de nombreux aléas climatiques tendent à réduire l'efficacité de ces systèmes. Des travaux collaboratifs entre ingénieurs, automaticiens, physiciens et biologistes devront être soutenus dans ce domaine en fort développement, ils conduiront à des progrès considérables. 


\section{Figures:}

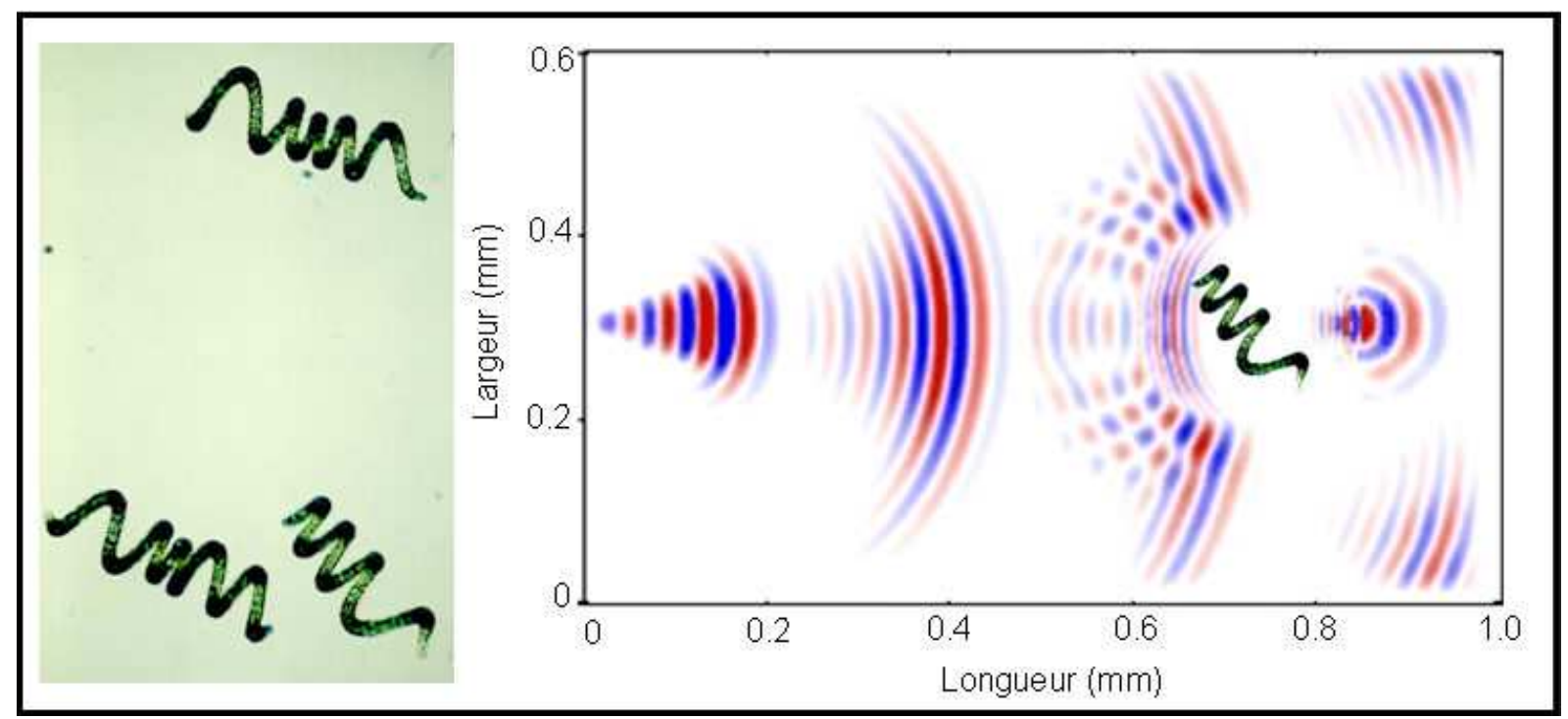

Figure 1 : A gauche : photographie de la cyanobactérie Arthrospira platensis (Spiruline). A droite : illustration de la diffusion d'une onde électromagnétique visible par le micro-organisme calculée par la méthode FDTD (Finite Difference, Time Domain ; pas de temps environ 1 picoseconde). On déduit de ces calculs les propriétés radiatives de la micro-algue (coefficients d'absorption et de diffusion ; fonction de phase angulaire). Compte tenu des indices de réfractions des microalgues, l'essentiel de l'énergie diffusée est contenue dans un angle de $+/-20^{\circ}$ autour de la direction incidente.

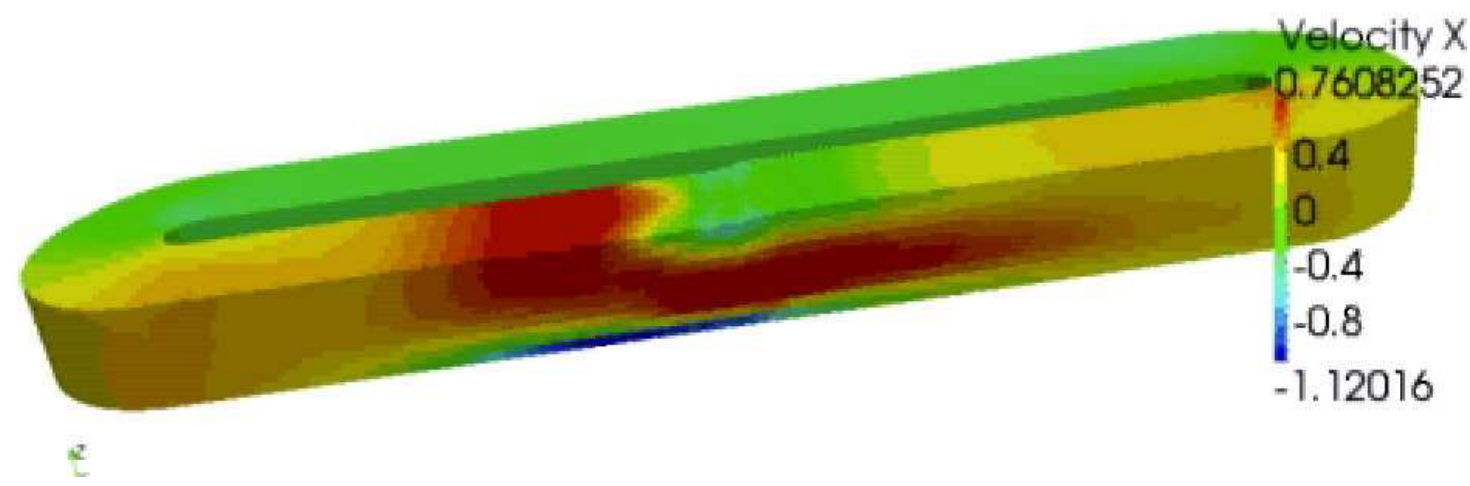

Figure 2 : Simulation numérique de l'écoulement dans un système de culture de type raceway, agité par une roue à aubes. Ces simulations permettent d'estimer la quantité de lumière perçue par chaque microalgue au cours du temps. 


\section{Références :}

PRUVOST J., CORNET J.-F., LE BORGNE F., JENCK J. 2011. Production industrielle de microalgues et cyanobactéries. Dans : Techniques de l'Ingénieur, rubrique Innovations (in200).

CADORET, J.-P. et BERNARD, O. 2008. La production de biocarburant lipidique avec des microalgues : promesses et défis. Journal de la Société de Biologie, 202 (3), 201-211.

\section{Glossaire :}

Raceway : canal ouvert à circulation de fluide (mis en mouvement par une roue à aubes) en forme de « 0 » (rappelant un champ de course).

Méthodes de Monte Carlo intégrales: méthodes numériques statistiques, basées sur un grand nombre de tirages aléatoires (représentant ici physiquement le trajet de chaque photon individuel). Les grandeurs physiques observables sont déduites de l'intégration de tous les chemins et évènements observés dans le volume. 